Trunk, M., Teichert, J. F.; Thomas, A.

\title{
Room-Temperature Activation of Hydrogen by Semi-immobilized Frustrated Lewis Pairs in Microporous Polymer Networks
}

Journal article | Accepted manuscript (Postprint)

This version is available at https://doi.org/10.14279/depositonce-8330

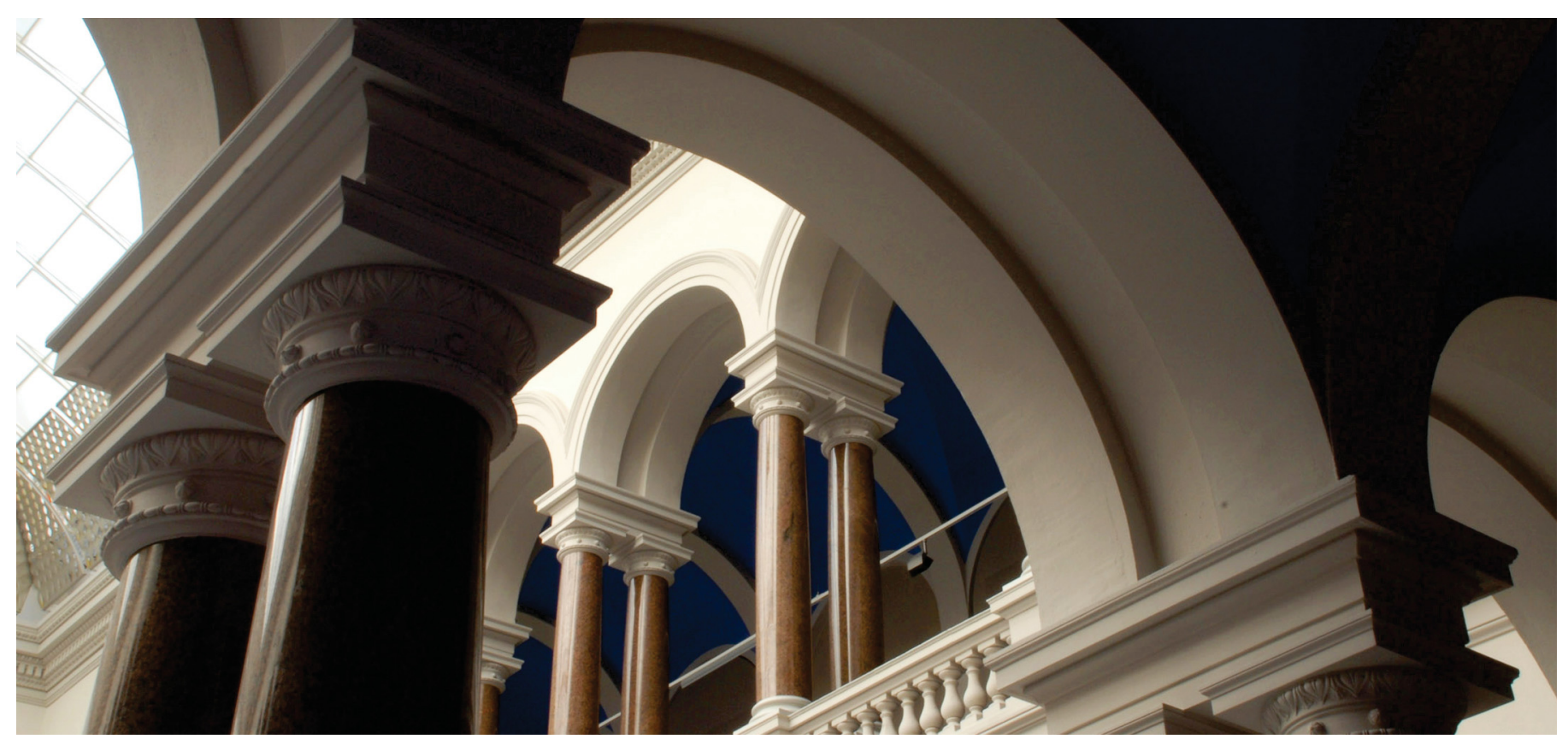

Trunk, M., Teichert, J. F., \& Thomas, A. (2017). Room-Temperature Activation of Hydrogen by Semiimmobilized Frustrated Lewis Pairs in Microporous Polymer Networks. Journal of the American Chemical Society, 139(10), 3615-3618. https://doi.org/10.1021/jacs.6b13147

This document is the Accepted Manuscript version of a Published Work that appeared in final form in the Journal of the American Chemical Society, copyright (c) American Chemical Society after peer review and technical editing by the publisher. To access the final edited and published work see https://pubs.acs.org/doi/10.1021/jacs.6b13147. 


\title{
ROOM-TEMPERATURE ACTIVATION OF HYDROGEN BY SEMI- IMMOBILIZED FRUSTRATED LEWIS PAIRS IN MICROPOROUS POLYMER NETWORKS
}

\author{
Matthias Trunk, ${ }^{\dagger}$ Johannes F. Teichert, ${ }^{\ddagger}$ and Arne Thomas*, ${ }^{\dagger}$ \\ †Department of Chemistry, Functional Materials, Technische Universitat Berlin, Hardenbergstraße 40, 10623 Berlin, Germany \\ $\ddagger$ Department of Chemistry, Organic Chemistry/Sustainable Synthetic Methods, Technische Universitat Berlin, Straße des 17. \\ Juni 115, 10623 Berlin, Germany
}

\begin{abstract}
Porous polymer networks based on sterically encumbered triphenylphosphine motifs, mimicking the basic sites employed in frustrated Lewis pair (FLP) chemistry, were synthesized via Yamamoto polymerization and their interactions with the strong Lewis acid $\mathrm{B}\left(\mathrm{C}_{6} \mathrm{~F}_{5}\right)_{3}$ probed. The combinations yield semi-immobilized FLPs, which are able to cleave dihydrogen heterolytically at ambient temperature and low hydrogen pressure.
\end{abstract}

Over the past decade, microporous polymer networks have garnered attention as materials for a wide variety of applications. ${ }^{1,2}$ Emanating from rigid organic building blocks, materials with very small pores between 0.5 and 2 nm and accessible surface areas of up to $6400 \mathrm{~m}^{2} \mathrm{~g}^{-1}$, as well as high thermal and chemical stability, ${ }^{3}$ have been synthesized by a number of polymerization methods, such as metal-mediated homocoupling reactions, metal-catalyzed cross-coupling reactions, condensation reactions, and many others. ${ }^{2,4}$ In contrast to metal-organic frameworks and covalent organic frameworks, these materials do not exhibit crystallinity; emerging from $\mathrm{C}-\mathrm{C}$ bond-forming reactions, they can forego the use of heteroatoms entirely, allowing for a pure hydrocarbon skeleton. This feature is especially advantageous when designing catalysts with highly sensitive active sites, as it enables the exclusion of nonorthogonal functional groups.

Such types of functional groups can be found as catalytic sites in frustrated Lewis pairs (FLPs). ${ }^{7}$ The members of this emerging class of molecular catalysts are solely based on maingroup elements and in most cases consist of a boron-based Lewis acid (LA) and a Lewis base (LB), usually comprising phosphorus or nitrogen as basic sites. ${ }^{8}$ Both active sites are sterically encumbered by bulky moieties in order to prevent them from forming a strong covalent bond and consequently quenching their opposing reactivities. Small molecules $\left(\mathrm{H}_{2}, \mathrm{CO}_{2}\right.$, etc.) fitting in between these bulky groups can be activated through double polarization by the acidic and basic sites, leading to (zwitter)ionic products (see Scheme 1). ${ }^{6,79}$ For this activation to take place, the steric hindrance must not be sufficient to prohibit any interaction; in solution, a weak but reversible coordination must be possible, lest the polarization be precluded entirely. ${ }^{10}$ Subsequently, the activated intermediate(s) can be used for reactions with a number of substrates.

The most intensely researched application for this type of catalyst is the heterolytic splitting of dihydrogen and its

Scheme 1. (Top) Equilibrium State of a Generic Frustrated Lewis Pair (LA = Lewis Acid, LB = Lewis Base) and Activation of Carbon Dioxide and Hydrogen; (Bottom) (Non)reversible Activation of Hydrogen by Reported FLPs5,6
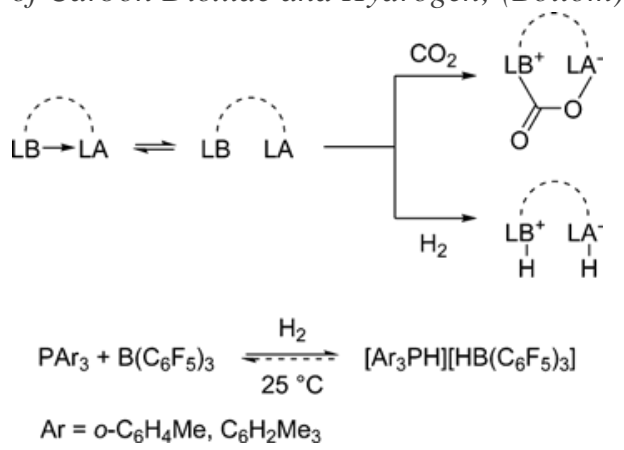
subsequent use in the reduction of $\mathrm{C}=\mathrm{C},{ }^{11} \mathrm{C}=\mathrm{N},{ }^{12}$ and $\mathrm{C}=\mathrm{O}^{13}$ double bonds as well as cleavage of silylenol ethers, ${ }^{14}$ among others. LA and LB can be part of the same molecule; i.e., both intra- and intermolecular FLPs are capable of activating small molecules. ${ }^{9}$ In many cases, though, the mechanistic properties of an activation process are deceptively simple. Even in cases of both active sites within the same molecule, theoretical studies rather suggest intermolecular activation via a two-fold head-totail interaction between two intramolecular FLP molecules. ${ }^{15}$

Whereas a great number of Lewis basic polymers has been published, since these are easy to obtain and often display interesting $\mathrm{CO}_{2}$ adsorption properties, only a handful of Lewis acidic moieties in porous polymers have been reported ${ }^{16,17}$ none of which are used for catalytic applications. Due to the use of very strong LAs such as tris(pentafluorophenyl)borane (commonly abbreviated to BCF) or related compounds, these catalysts are often highly sensitive to external factors such as humidity or oxygen, which makes their handling challenging. To overcome this issue, extensive studies toward electronic fine-tuning have produced robust LAs which can be handled without the use of inert gas techniques. ${ }^{18}$ Recent advances have furthermore afforded so-called "inverse" FLPs based on a weak and more robust LA but which are used in conjunction with a very strong and sensitive LB on the other hand. ${ }^{19}$

In order to be able to enhance stability and recyclability and to further increase the appeal of FLPs, the incorporation of FLPs into solid materials, e.g., a microporous polymer network, seems an advantageous endeavor. In this work we focused on the generation of FLPs emerging from porous polymers comprising sterically encumbered phosphine moieties, which are impregnated with a readily available strong LA. We demonstrate the ability of these semi-immobilized FLP systems to activate dihydrogen at ambient temperature and low hydrogen pressure.

The syntheses of the phosphine polymers were carried out according to the synthesis procedure for porous polymer networks via Yamamoto polymerization ${ }^{20}$ starting from tris(pbromophenyl)phosphine derivatives (see Scheme 2) with

Scheme 2. Syntheses of Triarylphosphine Polymersa
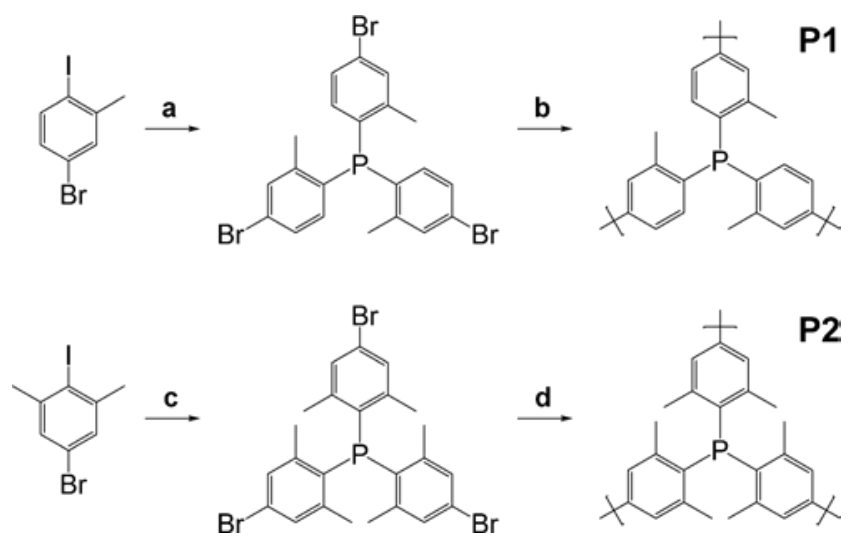

aReagents and conditions: (a) i-PrMgCl, THF, $0{ }^{\circ} \mathrm{C}, 1 \mathrm{~h}$, then PCl3, THF, $-78{ }^{\circ} \mathrm{C}$ to $\mathrm{rt}, 12 \mathrm{~h}$; (b) Ni(COD)2, DMF/THF, rt, $15 \mathrm{~h}$; (c) nBuLi, Et2O, $-78{ }^{\circ} \mathrm{C}, 2 \mathrm{~h}$, then PCl3, Et2O, $-78{ }^{\circ} \mathrm{C}$ to rt, $12 \mathrm{~h}$; (d) Ni(COD)2, DMF/THF, rt, $22 \mathrm{~h}$.

increasing steric demand around the phosphorus atoms. The structure of unmethylated triphenylphosphine polymer has been described previously. ${ }^{21,}{ }^{22}$ Due to the use of the less reactive aryl chloride starting material, harsher synthesis conditions were required, whereas in this work a milder synthesis $\operatorname{protocol}^{23}$ was applied due to the exclusive use of the more reactive brominated species.

Purification via Soxhlet extraction with methanol and drying in vacuo at $80{ }^{\circ} \mathrm{C}$ yields the product networks (Figure S1). Tris(2-methylphenyl)phosphine polymer P1 was collected as white and light solid, whereas tris(2,6-dimethylphenyl)phosphine polymer P2 has an intense yellow color and fluffy texture. P1 and P2 feature surface areas of 1045 and $947 \mathrm{~m}^{2} \mathrm{~g}^{-1}$, respectively, with P2 featuring a remarkable hysteresis (see Figure 1). These results are in line with the reported surface area of unmethylated triphenylphosphine polymer of $1284 \mathrm{~m}^{2} \mathrm{~g}^{-1} .{ }^{21}$ The decrease in surface area can thus be interpreted as a result of the increased monomer weight and pore blocking by increasing amounts of methyl groups.

Organic solvents such as toluene, bromobenzene, dichloromethane, and THF are readily absorbed by the polymers, which exhibit strong swelling properties and form gel-like mixtures with these solvents. To probe the properties of the synthesized materials as LBs, their behavior toward LAs such as commercially available BCF has to be examined. When a substance is predissolved in the absorbed solvent, it can be introduced into the pores of the polymer. Choosing a volatile 


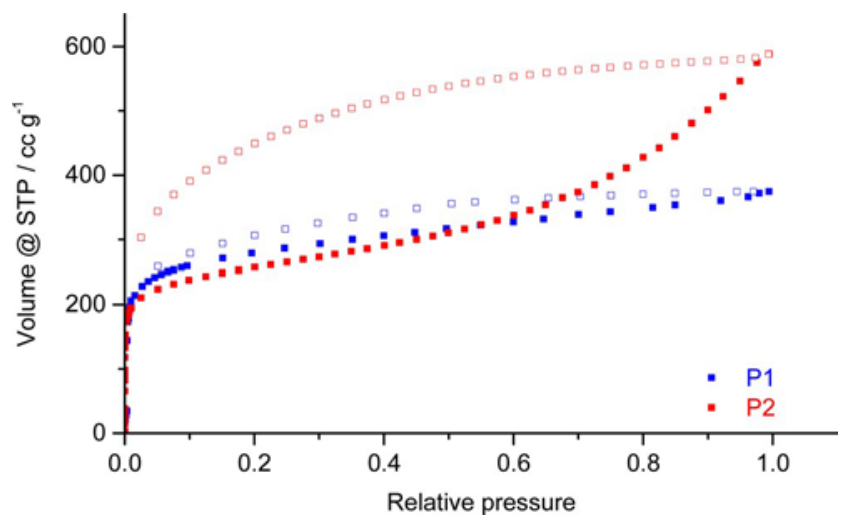

Figure 1. Nitrogen sorption isotherms of P1 (blue) and P2 (red).

solvent facilitates drying of the obtained material. BCF is well soluble in aromatic and chlorinated solvents. To gain information on the interaction between BCF as a LA and the bulky LB, no other Lewis acidic or basic functions may be present. Therefore, inside the glovebox, BCF and porous phosphine polymer were suspended in DCM, which is volatile, non-coordinating, and neither acidic nor basic, readily dissolves BCF, and leads to rapid swelling of the polymer. When impregnated with BCF solutions in non-coordinating solvents, e.g., DCM, the LA is readily absorbed into the polymer. The impregnation of the yellow polymer network P2 was accompanied by darkening of the color, while colorless P1 remained colorless. Washing with coordinating solvents such as diethyl ether or THF restores the original network. Even weakly coordinating solvents such as methanol or acetone are coordinated preferentially over the bulky phosphine moieties.

Both polymers (see Figure 2) as well as their composites with BCF exhibit strong fluorescence under UV irradiation (bottom row) at 254 (weak) and $366 \mathrm{~nm}$ (strong). BCF is readily absorbed by the polymer and quenches the fluorescence (see Supporting Information), especially for polymer P1,

P1

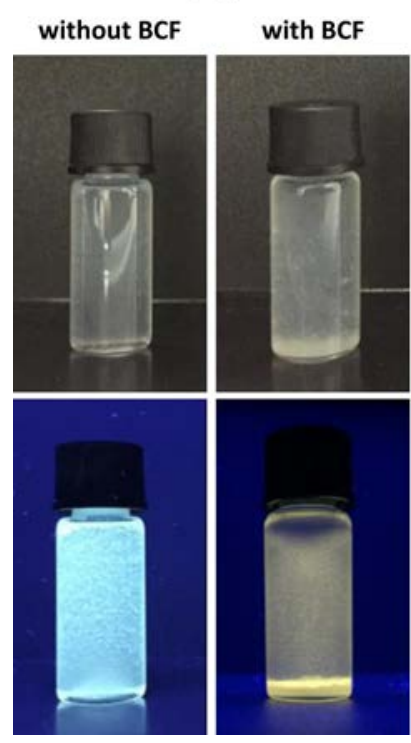

P2

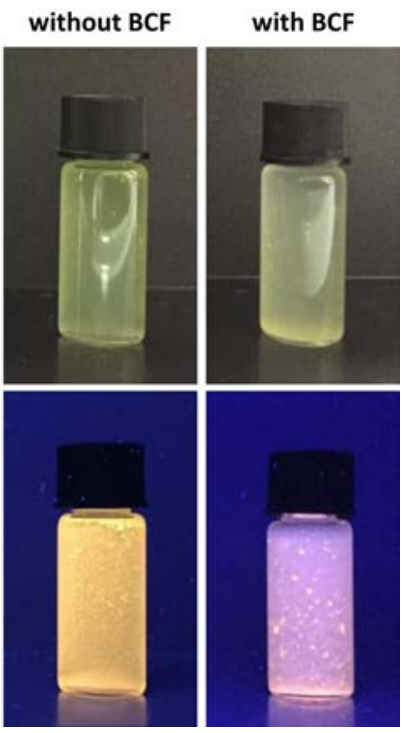

Figure 2. P1 (left block) and P2 (right block) in DCM suspension before and after addition of BCF. Top row, UV lamp off; bottom row, under irradiation of UV light $(366 \mathrm{~nm})$.

pointing to a stronger interaction with the LA (Figure S1). Furthermore, after addition of BCF, the finely dispersed polymer particles contract and sediment within a matter of minutes, whereas the empty polymer particles tend to collect at the surface of DCM.

Solid-state magic angle spinning NMR spectroscopy of the dried solids confirms the assumed structures (see Figure 3 and 


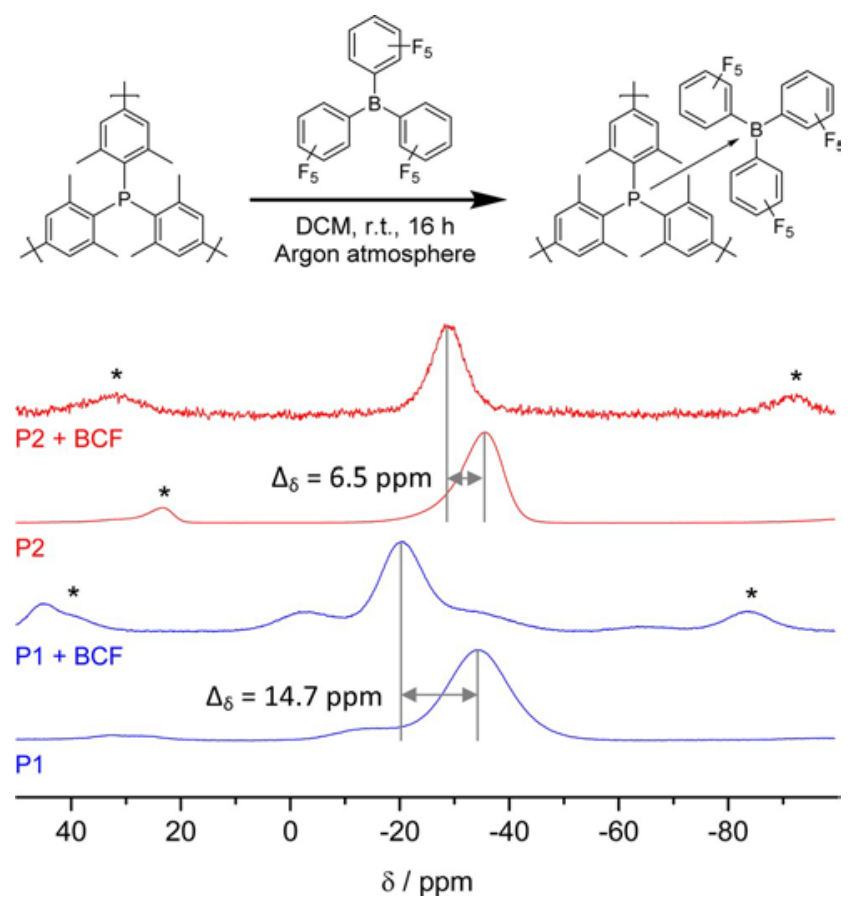

Figure 3. Impregnation procedure of triphenylphosphine derivative polymers (exemplified for P2) followed by 31P NMR spectroscopy: P1 (blue) and P2 (red) before (upper spectra) and after (lower spectra) impregnation with BCF. Asterisks denote spinning sidebands.

Figure S2). The ${ }^{31} \mathrm{P}$ NMR spectra each exhibit one main peak in the region of -34 and -36 ppm alongside a small impurity at approximately $+32 \mathrm{ppm}$, indicative of small amounts of phosphine oxide present in each network. These traces of phosphine oxide have been reported previously ${ }^{21}$ and seem to be the result of a side reaction of the Yamamoto polymerization, since the polymers were synthesized under rigorously inert conditions. Additionally, P1 and P2 are prone to phosphine oxide formation in air at elevated temperatures over elongated periods of time and should therefore be stored under inert gas.

Upon impregnation of the polymers with BCF and subsequent solvent removal, the ${ }^{31} \mathrm{P}$ solid-state NMR signals are shifted to lower field, as expected when coordinating to an acidic species. It is noteworthy that nearly the entire signal of the phosphine within the polymers is shifted downfield, showing that almost all phosphine groups in the polymer networks P1 and P2 are accessible to the molecular LA. Notably, the extent of the shift strongly correlates with the degree of methylation of the triphenylphosphine derivative. For P1 a shift of 14.7 ppm is observed, whereas for P2 a shift of only 6.5 ppm is observed (see Figure 3). This shift difference indicates that the bond strength decreases with increasing steric hindrance around the central phosphorus atom, which means that the higher the degree of frustration is, the weaker the emerging bond is rendered. This observation is quite remarkable.

According to Heine et al., ${ }^{10}$ the formation of a FLP is usually not observed at ambient temperature in solution; i.e., the solution NMR spectra of the individual components do not differ from the spectra of stoichiometric mixtures. Due to the only weakly coordinating nature of the FLP, the favorable interactions gained from association of LA and LB are dominated by the entropic contribution of the dissociation process. In the solid state, on the other hand, after solvent removal, LA and LB are forced into and kept in a "not-sofrustrated" state, so that the interaction can actually be observed, and its strength is determined by the difference in the chemical shifts, $\Delta_{\delta}$.

To verify this hypothesis, we compared the chemical shifts of the solid-state NMR spectrum of a "dry" FLP, consisting of the molecular counterpart of P2, tris(2,6-dimethylphenyl)phosphine, and BCF to the solid-state NMR spectrum of the phosphine molecule on its own. As expected, in the "dry" FLP, the phosphorus signal is shifted downfield by ca. 10 ppm compared to the phosphine (see Figure S3).

According to the molecular compounds, ${ }^{5,6}$ the combinations P1/BCF and P2/BCF should form FLPs and be active toward the cleavage of dihydrogen. When used in combination with BCF, the molecular equivalent of P1, tris(o-tolyl)phosphine, has been reported to activate hydrogen reversibly at ambient conditions to form [ $\left.\left(0-\mathrm{C}_{6} \mathrm{H}_{4} \mathrm{Me}\right)_{3} \mathrm{PH}\right]\left[\mathrm{HB}\left(\mathrm{C}_{6} \mathrm{~F}_{5}\right)_{3}\right]$, whereas the molecular counterpart of $\mathrm{P} 2$, trimesitylphosphine, forms the hydrogen adduct $\left[\left(1,3,5-\mathrm{C}_{6} \mathrm{H}_{2} \mathrm{Me}_{3}\right)_{3} \mathrm{PH}\right]\left[\mathrm{HB}\left(\mathrm{C}_{6} \mathrm{~F}_{5}\right)_{3}\right]$, which remains stable even when exposed to temperatures of up to $150{ }^{\circ} \mathrm{C}$. To prove the hydrogen activation capability of our polymeric materials, we performed H/D isotope scrambling experiments typically employed to demonstrate FLP behavior in a new material ${ }^{24}$ for composites of BCF with P1, P2, and the molecular counterpart of P1, tris(o-tolyl)phosphine. To that end, inside a glovebox, a 
Young-type NMR tube was charged with a stoichiometric mixture of P1/BCF or P2/BCF and cyclohexane- $\mathrm{d}_{12}$, and the suspension was subjected to ultrasonication for $15 \mathrm{~min}$ to ensure sufficient intermixing of the poorly soluble BCF and the polymer. Subsequently, the tube was pressurized with an $\mathrm{H}_{2} / \mathrm{D}_{2}$ mixture (ca. 1:1 v/v) to 6 bar and analyzed via ${ }^{1} \mathrm{H}$ NMR spectroscopy beginning several hours after pressurization of the tube. In both cases, an emerging triplet can be observed at 4.54 ppm shortly after pressurization, indicating the formation of HD (see Figure 4). The control reaction comprising only porous phosphine polymer P2 and solvent under $\mathrm{H}_{2} / \mathrm{D}_{2}$ pressure did not result in any conversion to HD. Although the control reaction with BCF as the sole reactant gave the scrambling product, albeit at much lower rate than the BCF/P2 composite, it must be pointed out that it has been proven theoretically ${ }^{25}$ and experimentally under low hydrogen pressure ${ }^{5}$ that $\mathrm{BCF}$ alone is not active in the heterolytic cleavage of $\mathrm{H}_{2}$. In our case, residues of protic solvents attached to BCF or trace water on the glass surface might be responsible for providing a proton shuttle in the activation of hydrogen. Furthermore, if a composite of BCF and another highly microporous polymer network devoid of any functional groups (PPN-6, $4172 \mathrm{~m}^{2} \mathrm{~g}^{-1}$, also prepared by Yamamoto polymerization) is used, no hydrogen activation product can be detected. It seems, therefore, that the impregnation procedure of a polymer has a negating effect on the activity of BCF itself. This could be caused by the highly hydrophobic and nonpolarizable surface of the networks. It can
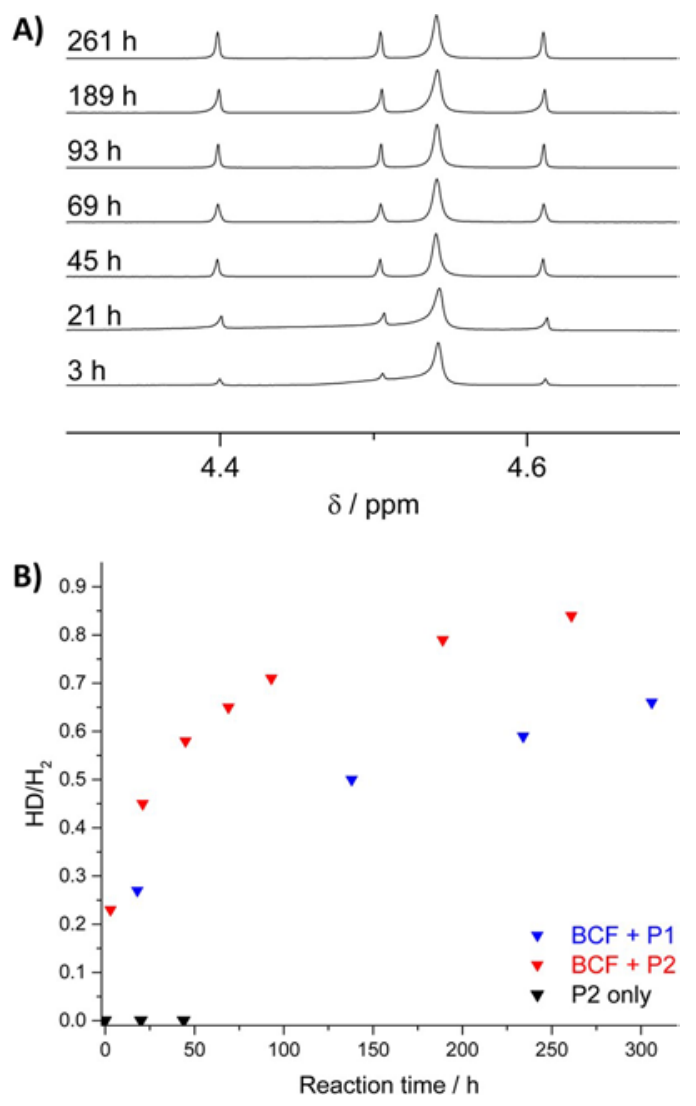

Figure 4. (A) Kinetic measurement of H/D exchange catalyzed be P2/BCF determined via $1 H$ NMR. (B) Integral ratio of HD/H2 plotted as a function of time.

therefore be assumed that the observed reactivity of the composites is not only independent of but inherently much higher than the activity of "pure" BCF. The composite tris(otolyl)phosphine/BCF gives the expected statistical $\mathrm{H}_{2} / \mathrm{HD}$ ratio after merely a few hours. This shows that the incorporation of the active sites into the polymer imposes a strong kinetic restriction on the H/D exchange process.

In summary, we hereby report the first synthesis of two semiimmobilized frustrated Lewis pairs with the basic components embedded into the backbones of microporous polymer networks. Addition of the commercially available Lewis acid, tris(pentafluorophenyl)borane, to polymers structurally akin to trimesitylphosphine yielded two functional heterogeneous hydrogen activation catalysts. Their ability to cleave dihydrogen at ambient temperature and low pressure was demonstrated via isotope scrambling experiments.

Further combinations of Lewis acidic and basic species need to be created to obtain heterogeneous catalyst systems capable of splitting dihydrogen at low pressures and to enable catalytic hydrogenation. Furthermore, full heterogenization of FLPs could even further enhance the reactivity and applicability of such systems. 


\section{ASSOCIATED CONTENT}

*S Supporting Information

The Supporting Information is available free of charge on the ACS Publications website at DOI: 10.1021/jacs.6b13147.

Experimental details on synthesis of monomers and polymers and hydrogen activation; ${ }^{13} \mathrm{C}$ NMR of polymers and molecular model compounds; fluorescence spectra and TGA of the polymers, including Figures S1- S4 (PDF)

\section{AUTHOR INFORMATION}

\section{Corresponding Author \\ *arne.thomas@tu-berlin.de \\ ORCID}

Johannes F. Teichert: 0000-0003-1043-8092

Arne Thomas: 0000-0002-2130-4930

Notes

The authors declare no competing financial interest.

\section{ACKNOWLEDGMENTS}

The authors thank Christina Eichenauer for surface area and TGA measurements. We also thank Douglas Stephan for his insights into the subject of FLP materials. This work was funded by the ERC Project ORGZEO (Grant No. 278593) and the DFG (Cluster of Excellence UniCat).

\section{REFERENCES}

(1) Thomas, A. Angew. Chem., Int. Ed. 2010, 49, 8328.

(2) Dawson, R.; Cooper, A. I.; Adams, D. J. Prog. Polym. Sci. 2012, 37, 530.

(3) Yuan, D.; Lu, W.; Zhao, D.; Zhou, H.-C. Adv. Mater. 2011, 23, 3723.

(4) Rose, M. ChemCatChem 2014, 6, 1166.

(5) Welch, G. C.; Stephan, D. W. J. Am. Chem. Soc. 2007, 129, 1880. (6) Ullrich, M.; Lough, A. J.; Stephan, D. W. J. Am. Chem. Soc. 2009, 131, 52.

(7) Welch, G. C.; Juan, R. R. S.; Masuda, J. D.; Stephan, D. W. Science 2006, 314, 1124.

(8) Stephan, D. W. J. Am. Chem. Soc. 2015, 137, 10018.

(9) Spies, P.; Erker, G.; Kehr, G.; Bergander, K.; Fröhlich, R.; Grimme, S.; Stephan, D. W. Chem. Commun. 2007, 2, 5072.

(10) Zeonjuk, L. L.; Vankova, N.; Mavrandonakis, A.; Heine, T.; Röschenthaler, G.-V.; Eicher, J. Chem. - Eur. J. 2013, 19, 17413.

(11) Greb, L.; Oña-Burgos, P.; Schirmer, B.; Grimme, S.; Stephan, D.

W.; Paradies, J. Angew. Chem., Int. Ed. 2012, 51, 10164.

(12) Spies, P.; Schwendemann, S.; Lange, S.; Kehr, G.; Fröhlich, R.; Erker, G. Angew. Chem., Int. Ed. 2008, 47, 7543.

(13) Lindqvist, M.; Sarnela, N.; Sumerin, V.; Chernichenko, K.; Leskela, M.; Repo, T." Dalton Trans. 2012, 41, 4310.

(14) Wang, H.; Fröhlich, R.; Kehr, G.; Erker, G. Chem. Commun. 2008, 45, 5966.

(15) Liu Zeonjuk, L.; St. Petkov, P.; Heine, T.; Röschenthaler, G.-V.; Eicher, J.; Vankova, N. Phys. Chem. Chem. Phys. 2015, 17, 10687.

(16) Suresh, V. M.; Bandyopadhyay, A.; Roy, S.; Pati, S. K.; Maji, T.

K. Chem. - Eur. J. 2015, 21, 10799.

(17) Li, Z.; Li, H.; Xia, H.; Ding, X.; Luo, X.; Liu, X.; Mu, Y. Chem. Eur. J. 2015, 21, 17355.

(18) Scott, D. J.; Fuchter, M. J.; Ashley, A. E. Angew. Chem., Int. Ed. 2014, 53, 10218.

(19) Mummadi, S.; Unruh, D. K.; Zhao, J.; Li, S.; Krempner, C. J. Am. Chem. Soc. 2016, 138, 3286.

(20) Schmidt, J.; Werner, M.; Thomas, A. Macromolecules 2009, 42, 4426. 
(21) Zhang, Q.; Yang, Y.; Zhang, S. Chem. - Eur. J. 2013, 19, 10024. (22) Pei, C.; Ben, T.; Guo, H.; Xu, J.; Deng, F.; Xiang, Z.; Cao, D.; Qiu, S. Philos. Trans. R. Soc., A 2013, 371, 20120312.

(23) Lu, W.; Yuan, D.; Sculley, J. P.; Zhao, D.; Krishna, R.; Zhou, H.-

C. J. Am. Chem. Soc. 2011, 133, 18126.

(24) vom Stein, T.; Perez, M.; Dobrovetsky, R.; Winkelhaus, D.; Caputo, C. B.; Stephan, D. W. Angew. Chem., Int. Ed. 2015, 54, 10178. (25) Nikonov, G. I.; Vyboishchikov, S. F.; Shirobokov, O. G. J. Am. Chem. Soc. 2012, $134,5488$. 